\title{
Google
}

\section{Hightech-Recherche im öffentlichen Web}

Rainer H. Bubenzer, Hamburg

NOTFALL \& HAUSARZTMEDIZIN 2004; 30: 268-270

Bei der Suche mit Google gibt es weitaus mehr Möglichkeiten zur optimierten Suche als zumeist bekannt. So können einzelne oder mehrere Begriffe, verbunden mit logischen Verknüpfungen, gesucht werden. Google gestattet es auch, bestimmte Wörter aus einer Suche auszuschließen oder bedeutungsähnliche Wörter zu suchen. Mittels Spezialparametern können alle Seiten eines speziellen Internetauftrittes gefunden werden, oder alle Seiten, die den gesuchten Suchbegriff im Titel oder dem Beschreibungstext der Hyperlinks enthalten. Auch alle Links auf eine bestimmte Website sind zu finden oder Websites, die inhaltlich dem gewählten Internetauftritt ähneln.

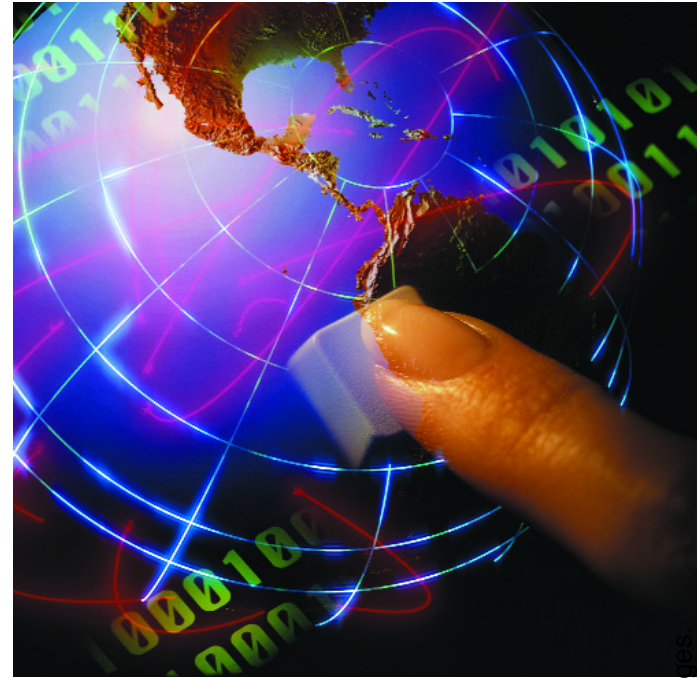

E twa 70-80\% aller Internet-Such- abfragen weltweit erledigt derzeit die US-amerikanische Suchmaschine Google (www.google.de oder www. google.com), deren Börsengang jetzt bevorsteht. Doch der Umgang mit der Suchmaschine Nr. 1 will gelernt sein und entwickelt erst durch Anwendung spezieller Parameter die hohe Leistungskraft einer modernen Datenbank. Der Preis dafür: Gesponserte Werbelinks, Google-Werbeeinblendungen auf vielen Websites und Akkumulation von Wissen über Surfer (Google beantwortet viele hundert Millionen Suchanfragen jeden Tag!). Die folgenden Hinweise umfassen wichtige Optionen der Google-Suche, weitere finden Sie auf den englischsprachigen Hilfeseiten des Anbieters (www. google.com/help/index.html).

\section{Normalsuche}

Ein einzelner Suchbegriff

in der Suchzeile von Google

Beispiel: Medizin. Ergebnis: 6,6

Mio. Fundstellen, zudem wird die Suchdauer ausgegeben (0,4 Sekunden). Die Ergebnisse werden nach einem Google-eigenen Verfahren angeblich nach 'Wichtigkeit' - sortiert (Google-Ranking). Oben und rechts auf der Ergebnisseite werden bezahlte Links („sponsored links“) angezeigt. Am Fuß der Seite können Sie zu den nachfolgenden Seiten kommen.

\section{Kombinationssuche} Mehrere Suchbegriffe ohne weitere Operatoren verknüpft (AND, +)

Google verknüpft diese Begriffe mit dem logischen Und-Operator, alternativ können Sie aber auch vor die Folgebegriffe ein + setzen oder Begriffe mit AND verknüpfen (unnötig). Möglich sind viele Dutzend Suchbegriffe gleichzeitig. Beispiel: Medizin Krankenhaus. Ergebnis: 387000 Fundstellen (= also einzelne Webseiten, auf denen beide Begriffe vorkommen).

\section{Mehrere Suchbegriffe mit}

Oder verknüpfen (OR, I)

Ein oder mehrere Suchbegriffe werden mit dem Oder-Operator verknüpft. Beispiel: Die Suche nach $M e$ dizin OR Krankenhaus ergibt zirka 2,5 Mio. Ergebnisseiten, die entweder einen oder beide Suchbegriffe enthalten.

\section{Geklammerte Suchtermini}

Und- und Oder-Operatoren können auch gemischt verwendet werden, am besten mit Klammerung. Beispiel: Arzt +Job + (Krankenhaus
OR Hospital) ergibt 33300 Suchresultate (alternativ zu „+“ können Sie auch AND verwenden).

Nicht-Operator (NOT, -)

Die stark vereinfachte LogikSyntax bei Google erlaubt trotzdem Verneinungen ( $=$ Boolscher NOTOperator). Beispiel: Die Suche nach Unfall -Auto ergibt alle Seiten, auf denen der Begriff Unfall vorkommt, nicht aber der Begriff Auto.

\section{Stop-Wörter}

Bei der Kombinationssuche verwendet Google keine so genannten Stop-Wörter, also kurze, sehr häufige Wörter wie in, auf oder Artikel. Um deren Verwendung zu erzwingen, ergänzen Sie das Wort mit einem vorangesetzten Pluszeichen.

\section{Termsuche}

"Suchterm1 Suchterm2“ findet den in Klammern eingeschlossenen Suchterm Mit den Anführungszeichen kann Google veranlasst werden, nach genau dem eingeschlossenen Suchterm zu suchen. Jedenfalls weitgehend genau, denn: Bindestriche, Leerzeichen und andere Satzzeichen zwischen zwei Begriffen werden ignoriert.

\section{Platzhalter/Wildcard * dient als Platzhalter für ganze Worte}

Der Platzhalter * für Worte ist eingeschränkt verwendbar, zum Beispiel findet Google mit „medizinische * " rund 2 Mio. Seiten mit Termini wie Medizinische Hochschule, Medizinische Dokumentation usw. Teilbegriffe können nicht gesucht 
werden, auch die Verwendung des Einzelzeichenplatzhalters ? ist nicht möglich.

\section{- Parameter (Tilde) \\ vor einem Suchterm findet bedeutungsgleiche Begriffe \\ Beispiel (in Kombination mit NOT, s.o.): Auto -Auto findet zirka 15 Mio. Seiten mit bedeutungsähn- lichen Worten aber nicht mit Auto. Also z.B. Automobil, Truck, Car, Ve- hicle.}

\section{Spezialsuchen \\ site:}

site:Siteangabe findet alle Seiten dieser Site (dieses Internetauftrittes).

Beispiel: site:www.thieme.de. Ergebnis: Von der Website des Thieme-Verlages sind 55900 von Google indiziert und über die Suchergebnis-Seiten zugreifbar.

inurl:

inurl:Suchterm findet alle Seiten, deren Name (URL - universal resource locator) den jeweiligen Suchbegriff enthält.

Beispiel: site:www.thieme.de inurl:notfallmedizin. Ergebnis: Diese Kombinationssuche findet alle 455 Seiten der Website des Thieme-Verlages, die in ihrem Namen den Begriff Notfallmedizin enthalten.

allinurl:

Wie inurl:, nur mit der Option, mehrere Begriffe anzugeben.

intitle:

Beispiel: intitle:Notfallmedizin findet alle Seiten, die im Titel (wird in der Kopfzeile des Browsers angezeigt) den Begriff Notfallmedizin enthalten.

\section{allintitle:}

wie intitle:, mehrere Suchbegriffe sind erlaubt.

\section{link:}

link:URL findet alle Seiten, die Hyperlinks enthalten, in denen das angegebene Sprungziel vorkommt.

Beispiel: link:http://www.spiegel. de. In diesem Beispiel verweisen rund 32800 Seiten auf die SpiegelHomepage.

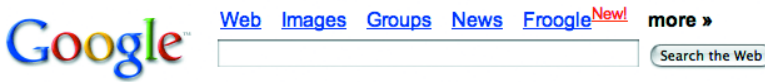

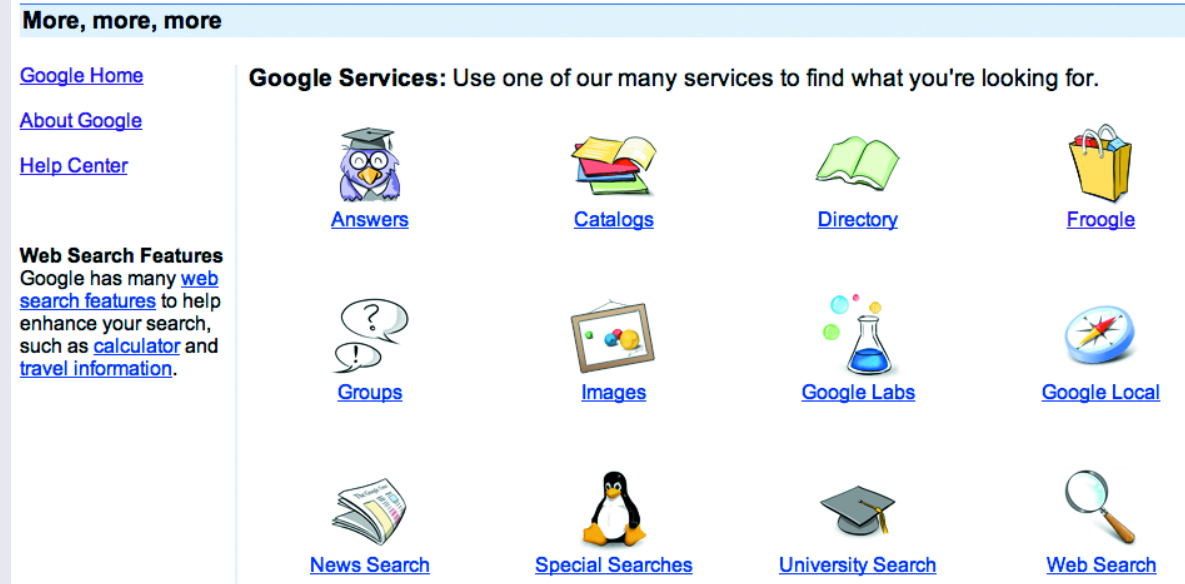

\section{inanchor:}

Beispiel: inanchor:Thieme-Verlag findet alle Seiten, die im Beschreibungstext von Internet-Verweisen (Hyperlinks) den Begriff ThiemeVerlag enthalten.

\section{allinachor:}

wie inanchor:, mehrere Suchbegriffe sind erlaubt.

\section{related:}

Beispiel: related:http://www. spiegel.de findet Seiten, die ähnlich sind wie Spiegel.de (was auch immer das nach Google-Entwickler Ansicht auch ist). In diesem Fall: $31 \mathrm{Si}-$ tes, von Focus über TAZ bis Chicago Tribune - also ebenfalls Zeitung beziehungsweise Zeitschriften.

\section{filetype:}

filetype:Dokumentenkürzel findet nur Dokumente mit dem entsprechenden Dateityp.

Beispiel: Die Google-Suche site:Thieme.de filetype:pdf gibt nur die 376 PDF-Dateien der ThiemeWebsite aus.

\section{intext:}

intext:Suchbegriff findet nur Text auf der Seite selbst (Hauptteil), nicht aber im Titel oder der URL.

\section{allintext:}

wie intext:, mehrere Suchbegriffe sind erlaubt.

\section{cache:}

cache:Suchbegriff durchsucht den Google-eigenen Zwischenspeicher (Cache).

Lesen Sie zuvor im Bereich Google-Spezialfunktionen (www. google.com/intl/de/features.html\# cached) alles nötige über GoogleCache.

\section{Hinweis}

Alle eingrenzenden Suchparameter können durch Vorstellung eines Minus-Zeichens auch verneint werden. Beispiel: Medizin -filetype: PDF verweist auf rund 3,4 Mio. Dokumente, in denen der Begriff Medizin vorkommt, die aber keine PDFDateien sind.

\section{Spielzeuge}

Geben Sie in der Suchzeile $420^{*} 1,1,6=$ ein, bekommen Sie das korrekte Ergebnis (Google-Taschenrechner). Auch kompliziertere Rechnungen inklusive Klammerung oder Exponentialrechnung sind möglich. Ebenso sind Umrechnungen von amerikanischen Maßen (z.B. inch, feet oder ton) ins metrische System möglich, zum Beispiel 23in= ergibt $58,42 \mathrm{~cm}$. Mit stocks:NYSE-Kürzel können Sie direkt die Yahoo-/Reuters-Kursdaten der New Yorker Börse einsehen, zum Beispiel stocks: BAY (Bayer Ag) oder stocks:EPC (Ep$\cos A G)$. Zahlenbereiche können durch Zahl1..Zahl2 gesucht werden, 
Nur Ihr Urteil bringt uns weiter!

\section{Wir wollen mehr \\ Informationsqualität für Sie.}

\author{
Helfen Sie mit, damit \\ Ihnen weiterhin eine \\ gute Fachpresse und ein \\ optimales Informations- \\ angebot zur Verfügung \\ stehen.
}

\section{In diesen Monaten befragt die Arbeits- gemeinschaft LA-MED Kommunikationsfor- schung im Gesundheits- wesen e.V. Sie und Ihre Kollegen zum Leseverhal- ten und zu Ihren Präfe- renzen in der Fachpresse.}

\section{Notfall}

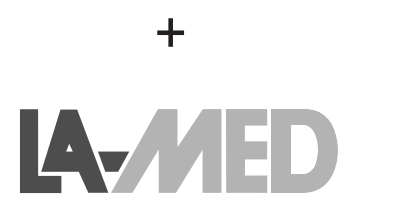

zum Beispiel findet Google beim Suchterm 1914..1918 Internetseiten, die sich zum Beispiel mit der Geschichte des ersten Weltkrieges beschäftigen.

\section{Suchseiten-Parameter}

Weitere Parameter können über „Einstellungen“ auf der GoogleSuchseite selbst definiert werden. Beispiele: Über 'Sprachtools' kann die Ergebnisausgabe gesteuert werden, zum Beispiel nur Resultate in einer bestimmten Sprache und/oder aus einem voreingestellten Land (entsprechend site:de = alle Seiten aus Deutschland). Die Option 'Übersetzen' sollen Sie besser ignorieren (außer Sie möchten ein wenig Unterhaltung: „Übertragung von Erregern“ = „transmission of exciters“). Die Option 'In den Ergebnissen suchen' schränkt die bereits durchgeführte Suche mit weiteren Begriffen ein (entsprechend der normalen Kombinationssuche mit zusätzlichen Begriffen). Die Option 'Erweiterte Suche' schließlich fasst viele der obigen Parameter zusammen und bietet zusätzliche zur Auswahl an, zum Beispiel die Anzahl von Ergebnissen pro Seite (10-100) oder die Ausgabe von Seiten, die in einem bestimmten Zeitraum aktualisiert worden sind (3, 6 oder 12 Monate).

\section{Google-Dienste}

Am häufigsten dürfte die vorgestellte Websuche benutzt werden. Doch Google hat im Laufe der Jahre fleißig verschiedenste Firmen aufgekauft, deren Informationen seither online teilweise weiter genutzt werden können. Beispiel: Der ehemalige DejaNews-Dienst, der die Diskussionsgruppen des kompletten Usenet indizierte, steht heute unter 'Google Groups' zur Verfügung (= UsenetSuchmaschine). Der Dienst 'Verzeichnis' ist hingegen eine minimal modifizierte Ausgabe von OpenDirectory (www.dmoz.org), dem weltgrößten, freien Internetseiten-Katalog. Der Suchmaschine Altavista abgeschaut ist der 'Bilder'-Dienst, der die Suche nach Fotos, Bildern und Grafiken im Internet erlaubt. Der 'News'-Dienst schließlich ist ein weitgehend automatisierter Nachrichtendienst, der aus unterschiedli- chen Internet-Quellen Informationen zusammenstellt. Den Schwerpunkt Gesundheit finden Sie unter news.google.com/news/de/de/ health.html. Ähnlich wie die aus deutscher Sicht illegale Speicherung von Website-Texten im Google-Zwischenspeicher („Cache“) ist auch dieser Dienst urheberrechtlich fragwürdig und aus journalistischer Sicht katastrophal. Für „smart shopper" in den USA steht der neue Produktsuchdienst www.froogle.com zur Verfügung, für bezahlte Anfragen der Dienst Google Answers (answers.google.com/answers/). 2,50 US-\$ pro Anfrage dürfte dem Tagesgehalt mindestens eines indischen Mitarbeiters entsprechen...

Die Google-Bar (Werkzeugleiste, Download: toolbar.google.com/intl /de/) klinkt ein Google-Suchfeld im Internet-Explorer ein, blockiert bei Bedarf die meisten unerwünschten Popup-Fenster und protokolliert alle Websites bei Google, die Sie beim Surfen besuchen (,gläserner Surfer“). „Google Zeitgeist“ (www. google.com/intl/de/press/zeitgeist.h tml) schließlich präsentiert häufige Suchtermini (im März am häufigsten war „Routenplaner“).

\section{Literatur}

1. Sixtus M. Google - Die dunkle Seite der Macht. De:Bug 71, 72, 73. 2004. www. de-bug.de/cgi-bin/debug.pl?what=show\& part=texte\&ID=2911 ff (Politische Manipulation mit Google)

2. Calishain T, Dornfest R. Google Hacks 100 Insider Tricks \& Tools. O’Reilly, aug. 2003. 368 Seiten, ISBN: 3897213621 (Fast alles über Google inklusive technischer Optionen für Webmaster)

\section{Anschrift des Verfassers}

Rainer H. Bubenzer

Medizin- und Wissenschaftsjournalist (DJV, KdM)

multi MED vision

Borselstraße 9

22765 Hamburg

Fax: 0 40/41 912877

E-Mail: Rainer@Bubenzer.com 